\title{
Amazing advances in the field of uro-oncology
}

\author{
Dora Niedersüß-Beke
}

After many years of stagnation, especially in bladder cancer, a new era has started with the approval of the checkpoint inhibitors in bladder cancer and renal cell carcinoma.

In this issue of $M E M O$, the most relevant topics in several fields of bladder and renal cell cancer have been summarized by several experts in the field.

In nonmuscle invasive urothelial cancer, promising new data show that checkpoint inhibitors after failure of BCG (Bacillus Calmette-Guérin) instillation help to avoid salvage radical cystectomies in up to $40 \%$ of patients. In the KEYNOTE-057 study, a phase II trial using pembrolizumab in patients with high-risk bladder cancer, complete response rates of up to $40 \%$ at 3 months were seen, with a high rate of maintained response for more than 9 months. Other checkpoint inhibitors such as atezolizumab, durvalumab, and nivolumab as monotherapy or in combination with BCG are under investigation in phase II and III trials for BCG naive or instillation unresponsive patients.

Neoadjuvant chemotherapy, my personal hot topic in bladder cancer, is still underutilized with only about $20 \%$ use before radical cystectomy. Two prospective phase II trials, PURE, using pembrolizumab, and ABACUS, using atezolizumab, could help make the impossible possible. With only small differences in trial design and a good toxicity profile-similar to that seen in the metastatic setting-complete remission rates (CR) between $30-40 \%$, and up to $54 \%$ in PD-L1positive patients, were seen. Importantly, these CR

\section{Niedersüß-Beke ( $\bowtie)$}

Department of Oncology, Hematology and Palliative Care, Wilhelminen Hospital, Montleartstraße 37, 1160 Vienna, Austria

dora.niedersuess-beke@wienkav.at rates are higher than historical data using conventional chemotherapy.

All is different in geriatric patients with urothelial cancer: Since this is the patient group we see daily in our clinic, we need to increase our effort to treat them appropriately. Does everybody need a radical cystectomy? Which other treatment options can we offer? Trimodal therapy with repeated TURBs (trans uretral resection of the bladder) and radiochemotherapy is feasible in this patient group. But on the other hand less is more in multimorbid geriatric patients. Answers to many of these questions are discussed in the article by Madersbacher et al. [1].

Renal cell cancer (RCC) is "the star" in the field of uro-oncologic malignancies, with a rapidly evolving treatment landscape of 13 novel substance approvals during the past 12 years in the metastatic setting. Because of the variety of substances, choosing the right treatment for the right patient is ever more challenging.

A lot has happened not only in systemic therapy, but also in the field of surgery. Due to increased availability and better quality of imaging, an increasing number of tumors-now 74\%-are diagnosed at an early stage $(\mathrm{T} 1+\mathrm{T} 2)$. This has led to a paradigm shift towards partial nephrectomy (PN). Open, laparoscopic and robotic assisted techniques are valid surgical options for this approach, but there are no data assessing laparoscopic versus robotic PN. Some studies have suggested that minimally invasive approaches entail fewer complications, lower blood transfusion rates, and shorter hospital stays. Similarly, in the metastatic setting primary nephrectomy is not the standard of care anymore, as shown in the CARMENA trial. In advanced disease, there is, however, still a role for nephrectomy in well selected patients, who are fit and oligometastatic or for pa- 
tients with an excellent response to TKI (tyrosine kinase inhibitor) therapy.

In the first-line systemic treatment setting, where checkpoint inhibitor combinations (I-O [immuno oncology] plus TKI or I-O plus I-O) are mandatory, risk stratification based on MSKCC (memorial sloan kettering cancer center) or IMDC (international metastatic RCC database consortium) to identify the best therapy will not be sufficient for a truly personalized treatment approach in the future. For example, data for predicting clinical benefit to VEGF targeted agents by the identification of transcriptional signatures were already investigated in the IMmotion 150 and 151 trials: Angiogenic gene expression signatures, T-effector and T-myeloid gene expression profiles were predictive for variable outcomes to treatment with atezolizumab plus bevacizumab versus sunitinib. Unfortunately, biomarkers for checkpoint inhibitors are still lacking, whereby PD-L1 expression testing and the identification of tumor mutational burden are not recommended by any of the guidelines due to doubtful predictive value. Meanwhile, more validated and cost-effective prognostic markers can be identified by simple blood tests, e.g., an elevated neutrophil to lymphocyte ratio is associated with poor clinical outcome in several entities. More biomarkers are expected to be identified by better genomic characterization of tumors like seen in the Pan Cancer Genome Atlas. The validation and routine integration of biomarkers have highest priority in ongoing and future clinical trials.

Finally, how to treat patients after failing first-line therapy? As there are several standard combinations using I-O plus TKI or I-O plus I-O in this setting, the choice of second-line treatment can be a challenge. Clinical considerations such as duration and depth of response, severity of progression, past toxicities, and some retrospective data may help in guiding the decision. Prospective clinical trials in the second-line setting are urgently needed.

Coming to the end, a big thank you to all authors [1-8] for their great articles in this rapidly changing time of uro-oncologic malignancies.
Conflict of interest D. Niedersüß-Beke declares that she has no competing interests.

\section{References}

1. Madersbacher S. Management of nonmetastatic muscle invasive bladder cancer in the elderly. memo. 2019. https:// doi.org/10.1007/s12254-019-00547-2.

2. Brönimann S, Shariat SF, Hassler MR. Nonmuscle invasive urothelial cancer-Bacillus Calmette-Guérin instillation or checkpoint inhibitor immunotherapy? Memo. 2019. https://doi.org/10.1007/s12254-019-00527-6.

3. Hutterer GC, Pichler M. Advanced/metastatic urothelial carcinoma of the bladder and upper urinary tract. Memo. 2019. https://doi.org/10.1007/s12254-019-00532-9.

4. Brönimann S, D'Andrea D, Shariat SF, Gust KM. Neoadjuvant therapy in urothelial cancer-new therapies on the horizon. Memo. 2019. https://doi.org/10.1007/s12254019-00533-8.

5. Marszalek M. First-line treatment of metastatic renal cell carcinoma: current standard of care. memo. 2019. https:// doi.org/10.1007/s12254-019-00550-7.

6 . Vogl U. Second-line therapy in metastatic renal cell cancer-how do we treat after immuno-oncology drugs? memo. 2019. https://doi.org/10.1007/s12254-019-005454.

7. Terzic J, Bauernhofer T. Biomarkers in renal cell carinoma. Memo. 2019. https://doi.org/10.1007/s12254-019-005490 .

8. Lenart S, Berger I, Hartig N, Madersbacher S, Ponholzer A. Surgery in renal cell carcinoma-when, whom and how? Memo. 2019. https://doi.org/10.1007/s12254-019-005481 .

Publisher's Note Springer Nature remains neutral with regard to jurisdictional claims in published maps and institutional affiliations.

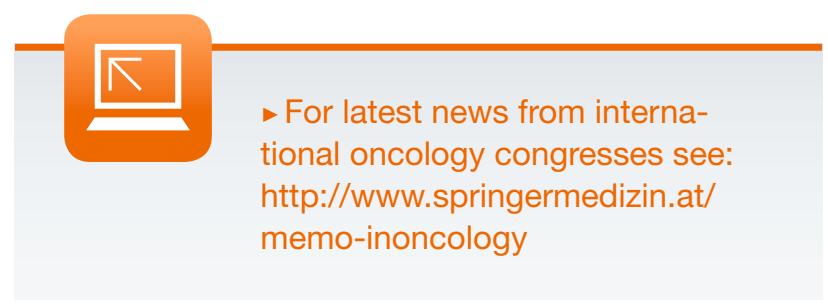

